\title{
Wachstumsbremse Wirtschaftsstrafrecht
}

\section{Problematische Folgen überzogener Steuerungsansprüche und mangelnder Randschärfe in der wirtschaftsstrafrechtlichen Begriffsbildung}

Prof. Dr. Hendrik Schneider

\section{Einführung}

Das Wirtschaftsstrafrecht wird weltweit nach dem Modell des politisch-publizistischen Verstärkerkreislaufs ${ }^{1}$ kontinuierlich ausgeweitet,

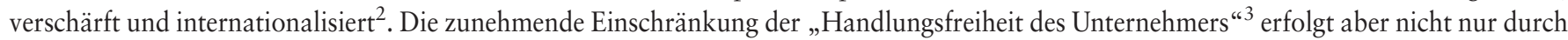
den Gesetzgeber, sondern auch durch die höchstrichterliche Rechtsprechung, die es versäumt, den konturenlosen Tatbeständen berechenbare Grenzen zu verleihen ${ }^{4}$. Insbesondere der Bundesgerichtshof hat sich offensichtlich von der allgemeinen „Korruptionshysterie“ anstecken lassen und sorgt nicht nur im Bereich der $\$ \$ 266,299,331 \mathrm{ff}$. StGB für Verunsicherung. Kritik formulieren hauptsächlich die am strafrechtswissenschaftlichen Diskurs teilnehmenden Verteidiger ${ }^{5}$, Compliance Officer ${ }^{6}$ und Verbandsjuristen ${ }^{7}$, die letztlich aber gezwungen sind, ihren Mandanten, Mitarbeitern und angeschlossenen Unternehmen die Leitlinien der höchstrichterlichen Rechtsprechung zu verdeutlichen und für den Arbeitsalltag handhabbar zu machen. Die punitive Kriminalpolitik im Gewand des modernen „prozeduralisierten“ Wirtschaftsstrafrechts ${ }^{8}$ beruht primär auf Richterrecht. Sie hat nur wenige potente Gegner aber viele Wegbereiter in Gestalt publizierender Staatsanwälte ${ }^{9}$ und Richter ${ }^{10}$, einiger Strafrechtslehrer ${ }^{11}$, einflussreicher Institutionen und nicht zuletzt auch der Kriminologie, die ihr kritisches Potential im Bereich des Wirtschaftsstrafrechts ${ }^{12}$ nie entfaltet hat ${ }^{13}$. Sukzessiv mehren sich Symptome eines anti-freiheitlichen und anti-marktwirtschaftlichen Wirtschaftsstrafrechts, das die Ökonomie behindert, unternehmensinterne Abläufe verlangsamt und Kosten verursacht ${ }^{14}$. Umkehr und Neubesinnung sind von Nöten:

2. Entwicklungstendenzen im Wirtschaftsstrafrecht

\subsection{Beispielhafte Verdeutlichung:} „Milestones“ aus der aktuellen Rechtsprechung

Die nachstehenden fünf Beispiele aus der aktuellen Rechtsprechung sind Meilensteine für die oben skizzierte Entwicklung, die durch zwei Charakteristika gekennzeichnet ist: Insbesondere die „Compliance Officer Entscheidung" des Bundesgerichtshofs und die „Vertragsarztentscheidung" des Oberlandesgerichts Braunschweig dokumentieren den zunehmenden „Steuerungsanspruch" der Gerichte, die sich nicht mehr darauf beschränken, Fälle zu entscheiden, sondern offensichtlich auch darauf abzielen, wirtschaftspolitisch als richtig empfundene Ergebnisse mit den Mitteln des Strafrechts durchzusetzen. Die beiden Vorlagebeschlüsse des 3. und 5. Strafsenats des Bundesgerichtshofs und die „Schulfotographenentscheidung " des 3. Senats geben darüber hinaus Aufschluss über die hierbei eingesetzten methodischen Prinzipien, die sich zunehmend von den etablierten Formen der Gesetzeshermeneutik lösen.

\subsubsection{Die „Compliance Officer Entscheidung" des 5. Strafsenats des Bundesgerichtshofs}

Die "Compliance Officer Entscheidung“ des 5. Strafsenats ${ }^{15}$ hat den wirtschaftsstrafrechtlichen Diskurs der Jahre 2009 und 2010 wie kaum ein anderes Urteil geprägt. Der Senat hatte über die Strafbarkeit eines Leiters der Innenrevision und Rechtsabteilung der Berliner Stadtreinigung zu entscheiden, der gegen die von dem Vorstand des Unternehmens zu verantwortenden Betrugstaten nicht eingeschritten war. Die über 60 Entscheidungsrezensionen und Besprechungsaufsätze beziehen sich allerdings nicht auf die tragenden Entscheidungsgründe, sondern auf einen kurzen Abschnitt der Urteilsbegründung, in dem der Senat strafrechtliche Berufsrisiken eines Compliance Officers aufzeigt:

„Eine ... neuerdings in Großunternehmen als "Compliance“ bezeichnete Ausrichtung, wird im Wirtschaftsleben mittlerweile dadurch umgesetzt, dass so genannte "Compliance Officers“ geschaffen werden ... . Deren Aufgabengebiet ist die Verhinderung von Rechtsverstößen, insbesondere auch von Straftaten, die aus dem Unternehmen heraus begangen werden und diesem erhebliche Nachteile durch Haftungsrisiken oder Ansehensverlust bringen können ... . Derartige Beauftragte wird regelmäßig strafrechtlich eine Garantenpflicht im Sinne des $\$ 13$ Abs. 1 StGB treffen, solche im Zusammenhang mit der Tätig- keit des Unternehmens stehende Straftaten von Unternehmensangehörigen zu verhindern. Dies ist die notwendige Kehrseite ihrer gegenüber der Unternehmensleitung übernommenen Pflicht, Rechtsverstöße und insbesondere Straftaten zu unterbinden .... ."

Die zitierte Passage ist für die Urteilsbegründung nur von marginaler Bedeutung. Sie dient allenfalls der Abgrenzung zwischen Tätigkeitsfeldern eines Leiters der Rechtsabteilung und eines Compliance Officers und stützt die Schlussfolgerung des Senats, der Angeklagte habe derart weitreichende Erfolgsabwendungspflichten nicht übernommen. Es handelt sich mithin um ein „obiter dictum", das häufiger in Entscheidungen des Bundesverfassungsgerichts, in Urteilen des BGH in Strafsachen aber bisweilen nur dann aufzufinden ist, wenn es sich um Fälle mit weitreichender politischer Dimension ${ }^{16}$ handelt.

\subsubsection{Die Vertragsarztentscheidung des} OLG Braunschweig

Dasselbe Stilmittel findet sich in einem vielbeachteten Beschluss des Oberlandesgerichts Braunschweig aus dem Jahr $2010^{17}$. Auch hier setzen sich über 30 Entscheidungsrezensionen und Besprechungsaufsätze kritisch mit einem obiter dictum im Beschluss des Oberlandesgerichts zu einem dogmatischen Detailproblem des \299 
StGB auseinander oder versuchen seine Konsequenzen für die Praxis auszuloten. In dem zur Entscheidung stehenden Fall hatte das Oberlandesgericht über die sofortige Beschwerde der Staatsanwaltschaft gegen den vorausgegangenen Nichteröffnungsbeschluss des Landgerichts zu entscheiden. Das Oberlandesgericht verwarf die sofortige Beschwerde, nahm das Verfahren jedoch zum Anlass, seine Rechtsauffassung zu der nicht entscheidungserheblichen Frage zu publizieren, ob ein niedergelassener Vertragsarzt als „Beauftragter der Krankenkasse“ als tauglicher Täter im Sinne des $\$ 299$ StGB in Betracht kommt:

„Nach Auffassung des Senats handelt es sich bei einem niedergelassenen Kassenarzt um einen Beauftragten i. S. d. genannten Vorschrift des geschäftlichen Betriebes der Krankenkassen. Ein Beauftragter in diesem Sinne ist, wer ohne Geschäftsinhaber oder Angestellter zu sein, aufgrund seiner Stellung berechtigt und verpflichtet ist, für den Betrieb zu handeln und auf die betrieblichen Entscheidungen Einfluss zu nehmen. Die Beauftragtenstellung eines Kassenvertragsarztes zeigt sich bereits in dem Rechtsverhältnis zwischen den Krankenkassen, den Kassenärzten, den Kassenpatienten und den Apotheken bei der Verordnung von Medikamenten, um die es hier geht. ..."

Die besondere Bedeutung des obiter dictums wird sogar noch dadurch unterstrichen, dass es in den Leitsatz der Entscheidung aufgenommen wird: „Bei einem niedergelassenen Kassenarzt handelt es sich um einen Beauftragten des geschäftlichen Betriebes einer Krankenkasse i. S. d. \299 StGB, soweit es um die Verordnung von Medikamenten geht“.

\subsubsection{Die Vorlagebeschlüsse des}

3. und 5. Strafsenats des

Bundesgerichtshofs vom 05. Mai bzw. 20. Juli 2011

Die genannten Beschlüsse der beiden Strafsenate beziehen sich ebenfalls auf die vom Oberlandesgericht Braunschweig thematisierte Frage der Rechtsstellung des Vertragsarztes ${ }^{18}$. Beide Senate sehen den Vertragsarzt nicht nur als Beauftragten der Krankenkasse im Sinne des $\$ 299$ StGB, sondern auch als Amtsträger im Sinne des $\mathbb{S} 11$ Abs. 1 Nr. 2c StGB an, wollen diese Rechtsauffassung aber offensichtlich durch eine Entscheidung des Großen Senats in Strafsachen dogmatisch „adeln“ lassen. Beide Beschlüsse betonen die besondere Praxisrelevanz der Entscheidung und die wirtschaftliche Tragweite im Zusammenhang mit dem intendierten Eingriff in Marketingstrategien der Medizinprodukteindustrie und begründen so ihre Ermessensentscheidung ${ }^{19}$, den
Großen Senat anzurufen. Dementsprechend heißt es in dem die Verordnung von Hilfsmitteln betreffenden Beschluss des 3. Senats:

„Die Beantwortung der Frage, ob ein niedergelassener, für die vertragsärztliche Versorgung zugelassener Arzt bei Wahrnehmung der ihm in diesem Rahmen übertragenen Aufgaben, hier konkret bei der Verordnung eines Hilfsmittels ... als Amtsträger im Sinne des $\int 11$ Abs. 1 Nr. 2 Buchst. c StGB handelt, ist von grundsätzlicher Bedeutung. Sie ist in der höchstrichterlichen Rechtsprechung bisher nicht geklärt und kann sich in einer Vielzahl von Verfahren erneut stellen. Ihre Beantwortung wirkt deshalb richtungsweisend für die Rechtsanwendung im Bereich der strafrechtlichen Verfolgung des sog. Pharmamarketing. ... Die Vorlage ist zur Fortbildung des Rechts erforderlich. Sie zielt auf die Festlegung neuer Auslegungsgrundsätze, als deren Folge sich ein geändertes Verständnis der Stellung des Vertragsarztes im Verhältnis zu den Krankenkassen ergibt.“20

Der 5. Senat schließt sich der Rechtsposition des 3. Senats an und subsumiert den Vertragarzt sowohl unter den Begriff des Beauftragten im Sinne des $\mathbb{} 299$ StGB als auch unter den Amtsträgerbegriff des $₫ 11$ Abs. 1 Nr. 2c StGB. Anstatt der prozessual möglichen aber „geräuschlosen“ Aussetzung des die Verordnung von Arzneimitteln betreffenden Verfahrens bis zur Entscheidung des Großen Senats entscheidet sich der 5. Senat ebenfalls für einen Vorlagebeschluss:

„Durch die unterschiedliche Fallkonstellation kann eine Vertiefung der dort zu erörternden Rechtsfragen erreicht werden. Dabei stimmt der Senat dem Ansatz des 3. Strafsenats zu, mit der Vorlage insbesondere im Bereich des Pharmamarketings zu einer einheitlichen, sich an entsprechenden Vorgaben des Großen Senats für Strafsachen orientierende Handhabung der Praxis zu gelangen."

\subsubsection{Die Schulfotographenent-} scheidung des 3. Senats des Bundesgerichtshofs vom 26. Mai 2011

Das letzte Beispiel zur Verdeutlichung der eingangs skizzierten Entwicklung ist die "Schulfotographenentscheidung" des 3. Senats, die sich ebenfalls auf das so genannte Korruptionsstrafrecht bezieht. Der Fall betrifft finanzielle Zuwendungen eines Schulfotographen u.a. an die Klassenkassen einer Schule, mit denen der Einsatz der Lehrkräfte im Zusammenhang mit der Organisation der Aufnahmen, der Entgegennahme und Weiterleitung des Geldes usw. entschädigt werden sollte. Der 3. Senat hält an der bisherigen Rechtssprechung fest, nach der ein Vorteil auch dann vorliegt, wenn es sich um eine Vergütung auf der Grundlage eines gegenseitigen Vertrages handelt ${ }^{21}$. Die hierdurch gegebene Aufweichung des Vor- teilsbegriffs und die Ausweitung der strafrechtlichen Verantwortlichkeit versucht der BGH sodann durch „prozeduralisierende“ Regelungselemente zu korrigieren, die in

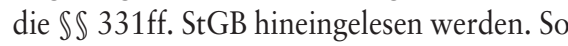
räumt der Senat zunächst ein, dass aufgrund der weiten Auslegung des Vorteilsbegriffs „nahezu jeder Vertragsschluss eines Amtsträgers in dienstlichen Angelegenheiten in Verbindung mit der dadurch begründeten Forderung sowie deren späteren Erfüllung “ einen Vorteil nach $\int \mathbb{S} 331 \mathrm{ff}$. StGB darstellt „und dies selbst dann, wenn es sich um einen im Rahmen der laufenden Dienstgeschäfte ordnungsgemäß geschlossenen Vertrag handelt" ${ }^{\prime 22}$.

Die notwendige Abgrenzung zwischen den Fällen „des unlauteren korruptiven Kaufs einer Diensthandlung im formellen Gewande eines gegenseitigen Vertrages“ und strafrechtlich unbedenklichen Fällen soll nach Auffassung des Senats über das Verwaltungsrecht erfolgen: „Als taugliches Abgrenzungskriterium kann hierbei die verwaltungsrechtliche Rechtmäßigkeit des Vertragsschlusses herangezogen und dabei insbesondere die Frage gestellt werden, ob die Diensthandlung in rechtlich zulässiger Weise von einer Vergütung abhängig gemacht werden darf". Da dies vorliegend nicht der Fall sei, und der Schule ein weitergehendes "Gebührenfindungsrecht“ nicht zustehe, sei die Aufbesserung der Klassenkassen als tatbestandsmäßiger Vorteil im Sinne der $\int \mathbb{S} 331 \mathrm{ff}$. StGB zu werten. Sind demgegenüber bei dem Vertragsschluss und den wechselseitigen Leitungserbringungen die Voraussetzungen des Verwaltungsverfahrens eingehalten, soll - ebenso wie bei der Drittmittelakquise des Hochschullehrers im Rahmen des jeweils geltenden Hochschulrechts $^{23}$ - auch das Strafrecht nicht zuständig sein.

\subsection{Kriminalpolitiker in Robe}

Die Fälle verdeutlichen zunächst einen wirtschafts- und kriminalpolitischen Steuerungsanspruch und ein entsprechendes Sendungsbewusstsein der zuständigen Richter. Urteils- oder Beschlussbegründungen haben grundsätzlich und ausschließlich die Funktion, den Tenor der Entscheidung zu rechtfertigen. Sie geben die Gründe für den Inhalt der Entscheidung an. Gemäß $\$ 13$ der Geschäftsordnung des Bundesgerichtshofs sind Tatbestand und Entscheidungsgründe deshalb „klar und möglichst kurz abzufassen. Sie sollen sich auf das Wesentliche und auf den Gegenstand der Entscheidung 
beschränken, auch entbehrliche Fremdwörter und nicht allgemein übliche Ausdrücke möglichst vermeiden“.

Diese gebotene „puristische“ Ausrichtung auf den Streitgegenstand bzw. die ermittelte Tat und die hiermit verbundene Selbstbeschränkung und Bescheidenheit, das geltende Recht lediglich auf einen Lebenssachverhalt anzuwenden, ist zumindest in den beiden zuerst genannten Beispielen nicht mehr ersichtlich. Sowohl das OLG Braunschweig als auch der 5. Strafsenat des Bundesgerichtshofs im Compliance Officer Fall wollen rechtspolitisch tätig sein und bestimmten kriminalpolitischen Vorstellungen zur Geltung verhelfen. Inhaltlich zielen die Richter - wie die oben angeführten Beispiele verdeutlichen - darauf $a b$, von ihnen als strafwürdig und strafbedürftig angesehene Marketinginstrumente der Pharmaindustrie und damit bestimmte Vorstellungen von der Funktionsweise des Gesundheitswesens durchzusetzen, die Verantwortung der Compliance Mitarbeiter eines Unternehmens für den übernommenen Aufgabenbereich zu schärfen oder (Schulfotographenentscheidung) eine wirtschaftliche Betätigung von Amtsträgern in die Schranken zu verweisen. Die Entscheidungsbegründungen der Urteile und Beschlüsse sind damit ein Akt der Rechtspolitik im Gewand der Rechtsprechung, durch den weitaus schneller und flexibler Veränderungen herbeiführt werden können, als durch den Gesetzgeber. Die obiter dicta sind das Stilmittel, durch das der kriminalpolitische Gestaltungswillen zum Ausdruck gebracht ${ }^{24}$ und der Entscheidungsauftrag verlassen wird ${ }^{25}$. Die Gerichte operieren folglich in der Funktion als akademische „Think Tanks“ und werden von den Normadressaten (z.B. Compliance Officern oder der Medizinprodukteindustrie) als „key opinion leader" wahr genommen ${ }^{26}$.

Obwohl ein obiter dictum keine Bindungswirkung entfaltet, erhält die Urteilsbegründung eine „Durchschlagskraft“, die in Ihrer Bedeutung eine wissenschaftliche Publikation bei weitem übertrifft ${ }^{27}$. Denn nur der Richter hat die „Definitionsmacht“ über die jeweilige Rechtsfrage. Das obiter dictum nimmt dem Normadressaten den Verbotsirrtum, macht „bösgläubig“ und zwingt zum Umdenken ${ }^{28}$. Auch die beiden Vorlagebeschlüsse des 3. und 5. Senats lassen sich in diesen Kontext einfügen. Die Vorgehensweise nach $\$ 132$ Abs. 4 GVG steigert die Spannung bei den Normadressaten weiter, unterstreicht die Wichtigkeit der Materie und die Tragweite des kriminalpolitischen
Anliegens. Der überflüssige zweite Vorlagebeschluss des 5 . Senats stärkt der dogmatischen Position des 3. Senats den Rücken und verengt die argumentativen Spielräume des Großen Senats, der nur mit großem Begründungsaufwand eine dogmatische Kehrtwendung vollziehen könnte.

\subsection{Methodische Prinzipien der Fortbildung des Wirtschaftsstrafrechts durch Richterrecht}

\subsubsection{Das Prinzip der weiten Auslegung so genannter „Auffangtatbestände“}

Bemerkenswert ist nicht nur das rhetorische Stilmittel, sondern insbesondere auch die methodische Vorgehensweise bei der Auslegung des geltenden Rechts, die insbesondere durch die Normstruktur des Wirtschaftsstrafrechts ermöglicht wird. Bereits mit der Einführung des Ersten und Zweiten Gesetzes zur Bekämpfung der Wirtschaftskriminalität war es das erklärte Ziel des Gesetzgebers, Beweisschwierigkeiten durch eine Korrektur des materiellen Strafrechts zu umgehen $^{29}$. Die Tatbestandsmerkmale der reformierten oder neu eingeführten Normen operieren daher mit einer im Hinblick auf das Bestimmtheitsgebot zumindest bedenklichen Lockerung der Gesetzesbindung ${ }^{30}$ und enthalten zwar „kernprägnante“, nicht aber „randscharfe“ 31 Rechtsbegriffe, die Exekutive und Judikative weite Beurteilungsspielräume zuerkennen. Ist es bei einzelnen Tatbestandsmerkmalen demnach kaum möglich, an „die Grenzen des möglichen Wortsinnes zu stoßen “ ${ }^{32}$, lassen sich mit ihnen unproblematisch und im Rahmen des juristisch „vertretbaren“ beliebige kriminalpolitische Vorstellungen dogmatisch adeln und umsetzen.

Besonders deutlich wird dies zum Beispiel bei dem Begriff des Amtsträgers im Sinne des $\int 11$ Abs. 1 Nr. 2c StGB und dem des Beauftragten eines geschäftlichen Betriebes ( $\mathbb{2} 299$ StGB). Beide Rechtsbegriffe haben die Aufgabe, den Täterkreis der als Sonderdelikte ausgestalteten Straftatbestände der SS 299f., 331ff. StGB zu definieren und innerhalb des Systemzusammenhangs der Normen die Funktion eines Auffangtatbestandes ${ }^{33}$. Durch die offene Formulierung der Tatbestandsvoraussetzungen (vgl. $\ 11$ Abs. 1 Nr. 2c StGB: ,sonstige Stelle“, „Aufgaben der öffentlichen Verwaltung “, bzw. \299 StGB: „Beauftragter“, „geschäftlicher Betrieb“) ist eine extensive Auslegung mög- lich und nach Auffassung zahlreicher Autoren und der Rechtsprechung zur Schließung von Strafbarkeitslücken sogar geboten ${ }^{34}$. Diese Rechtlage nutzen sowohl der 3. als auch der 5. Senat in den Vorlagebeschlüssen, um den Vertragsarzt, bei dem es sich nach überkommener Lesart noch immer um einen Freiberufler handelt, sowohl als Amtsträger als auch als Beauftragten der Krankenkassen einzustufen. Die gesetzlichen Krankenkassen sind danach einerseits „behördenähnliche Institutionen“ (\$ 11 Abs. 1 Nr. 2c StGB), andererseits aber auch „geschäftliche Betriebe“ im Sinne des $\$ 299$ Abs. 1 StGB. In Bezug auf ein und dieselbe Tätigkeit übt der Arzt daher vier Rollen aus. Er ist erstens Vertragspartner des Patienten, zweitens Unternehmer, der das wirtschaftliche Risiko des Praxisbetriebes trägt, drittens Beauftragter des geschäftlichen Betriebes Krankenkasse und viertens als Amtsträger dazu bestellt, für die Krankenkasse „Aufgaben der öffentlichen Verwaltung wahrzunehmen“.

\subsubsection{Das Prinzip der Prozeduralisierung}

Insbesondere die Schulfotographenentscheidung offenbart ein weiteres Charakteristikum der Auslegung wirtschaftsstrafrechtlicher Normen durch die Judikatur. Da die extensive Auslegung der interpretationsoffenen Rechtsbegriffe keine strafrechtsinternen Grenzen kennt, werden einschränkende Maßstäbe außerhalb des Strafrechts gesucht. Die einschlägigen Straftatbestände werden daher als Blankette interpretiert, wobei Verfahrensregelungen des Sozialrechts oder Verwaltungsrechts die Funktion von Voraussetzungen der Strafbarkeit übernehmen ${ }^{35}$. Für die Strafbarkeit des Schulfotographen bzw. Lehrers aber auch des Hochschullehrers bei der Einwerbung von Drittmittel ${ }^{36}$ oder des Arztes bei der Abrechnung von Leistungen der Gesetzlichen Krankenversicherung ${ }^{37}$ kommt es daher darauf an, ob er sich an das in außerstrafrechtlichen Normen, Verwaltungsverfügungen, Dienstvorschriften oder Satzungen geregelte Prozedere gehalten hat.

Durch diese Akzessorietät des Strafrechts wird Dynamik erzeugt und Wandel möglich, denn insbesondere Satzungen und Dienstvorschriften lassen sich unbürokratischer und schneller als Gesetze ändern und Strafbarkeitsrisiken daher elastischer als durch Strafgesetzgebung den als relevant empfundenen Bedürfnissen des Marktes anpassen. Andererseits verletzt diese In- 
terpretation des Strafrechts als Instrument verwaltungs- und zivilrechtsakzessorischer Wirtschaftslenkung aber elementare Grenzen des Strafrechts: Das Gebot der gesetzlichen Bestimmtheit der Straftatbestände (Art. 103 Abs. 2 GG) wird ausmanövriert, wenn Straftatbestände in Blankette uminterpretiert werden ${ }^{38}$. Denn soweit die Voraussetzungen der Strafbarkeit z.B. durch Dienst- und Verwaltungsvorschriften oder Satzungen abgesteckt werden, sind sie nicht mehr gesetzlich bestimmt im Sinne des Art. 103 Abs. 2 GG $^{39}$. Außerdem wird das ultima ratio Prinzip verletzt. Danach ist zwar eine „asymmetrische Akzessorietät“40 dergestalt denkbar, dass die strafrechtliche Verantwortlichkeit auf der Verletzung zivil- oder sozialrechtlicher bzw. verwaltungsrechtlicher Gebote beruht. Was sich nach den Maßstäben des Zivilrechts, Sozial- oder Verwaltungsrechts als rechtswidrig darstellt, ist aber nur dann strafbar, wenn der Gesetzgeber sich explizit für die Schaffung eines Straftatbestands entschieden hat (vgl. z.B. \266a StGB) oder das Verhalten unabhängig von der außerstrafrechtlichen Rechtslage unter den Tatbestand eines Strafgesetzes subsumiert werden kann.

Die mit der Idee der Lückenschließung legitimierte weite Auslegung interpretationsoffener Rechtsbegriffe und die Prozeduralisierung wirtschaftsstrafrechtlicher Normen durch deren systemwidrige Interpretation als Blankett stellt daher in mehrfacher Hinsicht einen Tabubruch dar. Er ist das Produkt übertriebener Hoffnungen in die systemsteuernde Kraft des Strafrechts und Ausdruck überzogener Steuerungsansprüche im Hinblick auf bestimmte sozial und wirtschaftspolitisch als richtig empfundene Zielvorstellungen.

\section{Kriminalpolitische Verstärker}

Die kriminalpolitischen Vorstellungen, die durch Richterrecht nach dem oben dargelegten modus operandi umgesetzt werden, dürften allerdings nur selten auf originären Vorstellungen der beteiligten Staatsanwälte und Richter beruhen, sondern sie assimilieren und artikulieren im gesamtgesellschaftlichen Diskurs bereits vorhandene Auffassungen und Strömungen. Um festzustellen, welchen wirtschafts- und kriminalpolitischen Vorstellungen durch Richterrecht zum Durchbruch verholfen wird, lohnt sich ein Blick auf die „Moralunternehmer" und „Normbenefiziare“, das heißt diejenigen Akteure des Wirtschaftslebens, die von einer Ausweitung der strafrechtli- chen Verantwortlichkeit profitieren und die sich kraft ihrer Definitionsmacht gegen die normunterworfenen Akteure durchsetzen können.

\subsection{Der Einfluss von Institutionen und Organisationen}

Unmittelbare Benefiziare ${ }^{41}$ der Ausweitung strafrechtlicher Verantwortlichkeit und der Intensivierung der Strafverfolgung im Bereich des Medizinstrafrechts sind vor allem die Gesetzlichen Krankenkassen, deren „Prüfgruppen Abrechnungsmanipulation“ Betrug und Korruption durch Vertragsärzte, Apotheker und Krankenhäuser nachgehen. Primäres Ziel ihrer Tätigkeit ist die Rückforderung von zu Unrecht erbrachten Leistungen der Gesetzlichen Krankenversicherung, bei der insbesondere das Strafrecht eine bedeutende Rolle spielt. Denn erstens verschafft der Vorwurf strafbarer Abrechnungsmanipulationen einen unschätzbaren Vorsprung bei den Verhandlungen mit den Leistungserbringern über die Rückerstattung erhaltener Vergütungen und zweitens sehen die verantwortlichen Mitarbeiter der Prüfgruppen und Stellen zur Bekämpfung von Fehlverhalten im Gesundheitswesen im Strafrecht ein wichtiges Präventionsinstrument, von dem sie sich Abschreckungseffekte erhoffen. Repräsentativ für diese Position ist der Erfahrungsbericht von Dina Michels ${ }^{42}$, die ihren Ärger über Einstellungsverfügungen der Staatsanwaltschaften kundtut und sich für die Sozialrechtsakzessorietät des Strafrechts und Lückenschließung im Korruptionsstrafrecht im Sinne der Rechtsprechung des OLG Braunschweig sowie des 3. und 5. Strafsenats ausspricht ${ }^{43}$. Es ist offensichtlich, dass die Verantwortlichen auf Seiten der Krankenkassen schon aufgrund ihrer beruflichen Tätigkeit sehr gut mit den zuständigen Staatsanwaltschaften vernetzt sind und im Rahmen der gesetzlich gebotenen Kooperation mit den Ermittlungsbehörden auch auf die Verfahrensabläufe Einfluss nehmen ${ }^{44}$.

Sehr aufschlussreich und kennzeichnend für die Rolle eines „Moralunternehmers“ ist weiterhin die Position von Transparency International (TI), einer international und in Deutschland seit 1993 in der Struktur eines eingetragenen Vereins operierenden Organisation, die ausweislich des Internetauftritts auf „strukturelle Veränderungen“ abzielt und sich insbesondere für die „Veränderung rechtlicher und politischer Rahmenbedingungen“ im „Kampf gegen Korruption“ einsetzt ${ }^{45}$. Im Rahmen der Arbeitsstrategie wird vornehmlich auf Netzwerkbildung gesetzt ${ }^{46}$. In der praktischen Arbeit treten Repräsentanten von TI außerdem auf zahlreichen Tagungen zum Thema Korruption und Korruptionsprävention auf und veröffentlichen Positionspapiere und Monographien, in denen die Thematik der Korruption z.B. durch Schätzungen über die Höhe der materiellen Schäden dramatisiert wird ${ }^{47}$, durch Verwendung eines diffusen Korruptionsbegriffs ${ }^{48}$ die Grenzen zwischen amoralischem und strafbarem Verhalten verschwinden und abweichende Meinungen, die sich für eine Zurücknahme des Strafrechts einsetzen, systematisch ausgeblendet werden. Das Strafrecht hat dabei die Funktion einer Speerspitze. Vorzugswürdig sind stets Auffassungen, die ein Maximum an Kriminalisierung zulas$\operatorname{sen}^{49}$. Parallel werden Gesetzesänderungen zur Erweiterung des Korruptionsstrafrechts gefordert und bestehende Vorschläge nach einer Ausweitung der strafrechtlichen Verantwortlichkeit unterstützt ${ }^{50}$. Es ist Teil der Netzwerkbildung von TI, diejenigen Richter und Staatsanwälte auf Tagungen einzuladen und Publikationsmöglichkeiten zu eröffnen, die für die in das Weltbild von TI passenden Entscheidungen verantwortlich sind ${ }^{51}$. Der „Ritterschlag“ durch TI symbolisiert den Akteuren dabei, auf der „richtigen Seite“ zu stehen, während abweichende Perspektiven zumindest faktisch als amoralisch, vertrauensunwürdig und undemokratisch ausgegrenzt werden.

Bei genauerem Hinsehen schwingen in den einschlägigen Publikationen aus der Feder der bis in die Bundespolitik hinein einflussreichen Repräsentanten von TI bisweilen antikapitalistische Ressentiments mit, die Rückschlüsse auf das gesellschaftliche Idealbild zulassen. In der „nicht-korrupten Demokratie“ ist es nach Auffassung von TI verdächtig, wenn Pharmaunternehmen mehr Geld für Marketing ausgeben, als für Forschung. Das Recht auf informationelle Selbstbestimmung tritt gegenüber dem Transparenzgebot zurück, Arbeitnehmerdatenschutz muss sich den Belangen der Korruptionsaufdeckung unterordnen ${ }^{52}$.

\subsection{Das Selbstverständnis der Kriminologie}

\subsubsection{Renaissance marxistischer Ansätze und rational choice Theoreme}

Das geschilderte Weltbild von TI deckt sich mit der Position bestimmter international 
einflussreicher Kriminologen, die ihrerseits im Anschluss an Klassiker der marxistischen Kriminologie $^{53}$ anti-marktwirtschaftliche Positionen vertreten und im Zusammenhang mit der Bekämpfung der Wirtschaftskriminalität auf ein starkes Strafrecht setzen. In den einschlägigen Arbeiten dominiert ein kriminalätiologischer Ansatz, nach dem die Ursachen für Wirtschaftskriminalität sowohl in den „Systembedingungen“ als auch im Individuum zu suchen sind. Die „Kultur des Wettbewerbs“ („culture of competition" ${ }^{\text {54 }}$ ), der „market society“ und die Versorgung der Märkte mit „Versuchungen“ („lure“ ${ }^{\text {55)}}$ ) werden als Ursache der Wirtschaftskriminalität ausgemacht und zum Ausgangspunkt eines Plädoyers für eine Veränderung des Wirtschaftssystems (Verbot bestimmter Produkte des Finanzmarktes, stärkere staatliche Regulierung, höhere Kontrollintensität) genommen. Dem Strafrecht soll dabei eine zweifache Funktion zukommen. Erstens soll es die Transaktionskosten der Straftat erhöhen und damit nach dem Modell der „crime as a choice“ 56 Theorie - die Abwägung des Individuums für und gegen die Wirtschaftsstraftat beeinflussen. Zweitens soll es ein moralisches Bollwerk gegen zügelloses Gewinnstreben darstellen und diejenigen wirksam stigmatisieren können, die seine Grenzen verletzen $^{57}$.

Der Ruf namhafter Kriminologen nach dem starken, eingriffsintensiven Wirtschaftsstrafrecht operiert mit Vorstellungen, die im Bereich der Elends- und Straßenkriminalität insbesondere durch die kritische Kriminologie und empirische Erkenntnisse zu den präventiven Effekten eingriffsintensiver Sanktionen längst verworfen wurden ${ }^{58}$. Dass sie im Zusammenhang mit der Verhinderung von Wirtschaftskriminalität eine Renaissance erfahren und Beachtung finden, wird durch die Weltwirtschafts- und Bankenkrise zwar nachvollziehbar, überzeugend sind die Ansätze hingegen nicht.

\subsubsection{Ausgeblendete kriminologische Gegenpositionen}

Empirische Untersuchungen $\mathrm{zu}$ den personalen und situativen Risikofaktoren der Wirtschaftskriminalität sprechen dezidiert gegen die Annahme eines rationalen Wahlverhaltens des Wirtschaftsstraftäters für oder gegen die Wirtschaftsstraftat. In der Untersuchung von Schneider\&John „Der Wirtschaftsstraftäter in seinen sozialen Bezügen “59 zeigte die Analyse der Tatgelegenheitsstrukturen bei den vier unterschied- lichen idealtypischen Konstellationen personaler Risikofaktoren („Täter mit einem wirtschaftskriminologischen Belastungssyndrom“, „Krisentäter“, „Abhängige“ und „Unauffällige“), dass langfristige Kosten der Straftat (Entdeckung und Sanktionierung der Tat zu einem späteren Zeitpunkt) nicht in Rechnung gestellt, bagatellisiert oder verdrängt werden. Die untersuchten Wirtschaftsstraftäter entsprachen daher gerade nicht dem Idealtypus des homo oeconomicus, weil nur der (erhebliche) kurzfristige Nutzen, nicht aber die Kosten - zum Beispiel auch in Gestalt der Sanktionshärte - in eine denkbare Bilanzierung einbezogen wurden.

$\mathrm{Zu}$ ähnlichen Ergebnissen kam die Untersuchung von Cleff, Luppold, Naderer und Volkert $^{60}$, die insbesondere bei den Täterprofilen der „Narzissten“ und der „Naiven" von einer geringen Ansprechbarkeit durch das Strafrecht und seine Sanktionen ausgehen. Der „Naive“ stolpere über die Gelegenheit, „ohne sie wirklich konsequent zu reflektieren, geschweige denn ihre Konsequenzen zu erfassen“, während der „Narzisst“ aus Selbstüberschätzung keine Konsequenzen fürchte ${ }^{61}$. In dieselbe Richtung weisen die Erkenntnisse der die Methode der Spieltheorie nutzenden experimentellen Wirtschaftswissenschaften, die kürzlich von Laue für die wirtschaftskriminologische Forschung fruchtbar gemacht wurden ${ }^{62}$. In den von Laue analysierten Spielsituationen orientierte sich kein Spieler am Prinzip der Nutzenmaximierung, sondern an Empfindungen von Fairness, Gerechtigkeit und Reziprozität. Menschen sind danach zumindest in den experimentellen Spielsituationen bereit, wirtschaftliche Nachteile zu erleiden, um andere fair und gerecht $\mathrm{zu}$ behandeln. Werden sie unfair behandelt (z.B. durch einen seinen eigenen Nutzen maximierenden Spieler), zeigen sie eine deutliche Präferenz dafür, dieses Verhalten zu sanktionieren, auch wenn ihnen die Sanktionierung nur wirtschaftliche Nachteile und keine Vorteile einbringt und sich deshalb aus der Perspektive des rational choice approach als ökonomisch unvernünftig erweist.

\section{4. Ökonomische Folgen}

Die geschilderte Entwicklung im Wirtschaftsstrafrecht unterliegt daher zahlreichen Einflussfaktoren und ist Teil einer gesamtgesellschaftlichen Strömung, in der dem Staat zunehmend die Rolle eines ,intervenierenden Sozialstaats“ zuerkannt wird. Das „regulative Recht“ ist das Mittel der „aktiven Steuerung“ und Verhaltensbeeinflussung und das Strafrecht das „Machtmedium “, durch das die erwünschten Ziele mit dem erforderlichen Nachdruck umgesetzt werden ${ }^{63}$. Die Umgestaltung und Verschärfung des Strafrechts durch die „weite Auslegung von Auffangtatbeständen “ und „Prozeduralisierung “ ist aber nicht nur dogmatisch bedenklich und kriminalpolitisch ineffizient, sondern auch kostenintensiv und Ressourcen vernichtend ${ }^{64}$. Die „Wachstumsbremse Wirtschaftsstrafrecht" zeigt sich insbesondere in folgenden Punkten:

\subsection{Die Überlastung der Strafrechtspflege und ihre Konsequenzen}

Werden die Schleusen des materiellen Rechts geöffnet, muss die Flut potentieller Straftaten durch die Strafrechtspflege und ihre Instanzen bewältigt werden. Dies ist ohne maßgebliche Aufstockung der personellen Ressourcen unmöglich und führt entweder zu einem inflationären Ausweichen auf das „soft law“ der $\mathbb{S} 153 \mathrm{ff}$. StPO oder zu einem exponentiellen Anstieg des Dunkelfeldes, weil die Kontrolle der potentiell verdächtigen Geschäftsvorfälle nicht durchführbar ist. Beide Varianten beeinträchtigen maßgeblich die Normanerkennung und den Glauben an die Legitimität der Strafgesetze und führen damit langfristig zu einer Erosion des Strafrechts auch im Kernbereich der Normen $^{65}$.

Erklärt z.B. die Rechtsprechung den Vertragsarzt zum tauglichen Täter eines Bestechungsdeliktes, steht jegliche Kooperation mit der Medizinprodukteindustrie unter Korruptionsverdacht. Dies gilt zum Beispiel für das besonders praxisrelevante Feld der „Anwendungsbeobachtungen“, die TI zu unrecht ${ }^{66}$ grundsätzlich verbieten möchte, und die gegenwärtig von rund $50 \%$ der etwa 140.000 niedergelassenen Vertragsärzte $^{67}$ für die Pharmaindustrie auf vertraglicher Grundlage durchgeführt werden. Da Anwendungsbeobachtungen auch Marketinginstrumente zur Vertriebsförderung der jeweiligen Arzneimittel darstellen, wären die häufig fremdsprachigen Verträge bei vorliegendem Anfangsverdacht nach Anhaltspunkten für das Vorliegen einer Unrechtsvereinbarung zu überprüfen. Dasselbe gilt für Sponsoringvereinbarungen von Fortbildungsveranstaltungen, im Hinblick auf die Kooperation niedergelassener Vertragsärzte mit Krankenhäusern usw. Die bisher bestehenden Einheiten der Staatsanwaltschaft und Polizei zur „Bekämpfung von Fehlverhalten im Gesundheitswesen“ 
müssten vor der zu erwartenden Verfahrensflut kapitulieren. Die Überlastung würde sich nicht zuletzt auch auf die Bearbeitung der schweren Erscheinungsformen der Kriminalität auswirken, für deren Erledigung ebenfalls nicht mehr die erforderlichen personellen Ressourcen zur Verfügung stünden.

\subsection{Kosten auf Unternehmensebene}

Auf Unternehmensebene ist mit einer inflationären Anwendung der so genannten Vorfeldverteidigung zu rechnen, für deren Vertrieb die unberechenbare und intransparente Rechtsprechung in Wirtschaftsstrafsachen ungewollt bereits jetzt das beste Marketinginstrument der auf Strafrecht spezialisierten Berater darstellt.

Im Rahmen der „Vorfeldverteidigung“ werden bestimmte Geschäftsabläufe im Vorfeld der Transaktion strafrechtlich begutachtet. $\mathrm{Da}$ die Entscheidungsträger in den Unternehmen häufig keine Juristen sind und in den Rechtsabteilungen nur selten auf Strafrecht spezialisierte Rechtswissenschaftler arbeiten, wird die strafrechtliche Prüfung in der Regel ausgelagert und auch aus Gründen der Haftungsdelegation Dritten überantwortet. Der externe Sachverstand wird ferner deshalb benötigt, weil das Strafrecht in seiner Auslegung durch die höchstrichterliche Rechtsprechung derart komplex ist, dass es von den Normadressaten nicht mehr überblickt und durchschaut werden kann. Die Grenzen zwischen erlaubter Geschäftstüchtigkeit und Straftat sind flüssig und die Expertisen der Strafjuristen sollen die Grenzen des noch Zulässigen ausloten und vor dem Zugriff des Staatsanwaltes schützen. Dies verursacht zunächst direkte Kosten, die für die Vergütung der externen Berater anfallen. Außerdem entstehen Sekundärkosten aufgrund der Verlangsamung der Geschäftsprozesse und der Reibungsverluste, die durch die Angst und Zögerlichkeit der Mitarbeiter verursacht werden, aufgrund der Unkenntnis der Grenzen des Strafrechts und ohne Absicherung durch ein externes Gutachten oder ein Votum der Compliance Abteilung folgenschwere Fehler zu begehen.

Darüber hinaus wird wiederum die Geltungskraft des Strafgesetzes beeinträchtigt. Da die Festlegung der strafrechtlichen Grenzen durch die höchstrichterliche Rechtsprechung von den Normunterworfenen häufig nicht nachvollzogen wird, verliert das Strafrecht seinen Charakter als sozialethisches Unwerturteil $^{68}$. Es scheint kaum mehr vom eigenen Geschick und der eigenen inneren Haltung zur Gesetzeskonformität, sondern vom Zufall abhängig zu sein, ob strafrechtliche Vorwürfe im Zusammenhang mit der Berufstätigkeit erhoben werden oder nicht ${ }^{69}$.

Aufgrund der dramatischen Folgen, die für Unternehmen und Individuen bereits mit einem Ermittlungsverfahren verbunden sind, führt das unberechenbare Strafrecht seine Benefiziare auch dazu in Versuchung, es für eigene monetäre Zwecke zu instrumentalisieren. Der Vorwurf, eine Straftat begangen zu haben, sichert die Verhandlungsposition im Rahmen einer arbeitsrechtlichen Auseinandersetzung mit einem unliebsam gewordenen Mitarbeiter oder er drückt den Preis beim Unternehmenskauf, wenn bei einer „integrity due diligence“ des Zielunternehmens möglicherweise strafrechtlich relevante Sachverhalte zu Tage getreten sind. Auch das „Anschwärzen“ des Wettbewerbers kann ein Mittel sein, um möglicherweise durch Straftaten entstandene Vorsprünge $\mathrm{zu}$ neutralisieren. Ökonomische Prozesse werden durch Strafrecht somit verzerrt und gegebenenfalls unlauter beeinflusst.

\section{Neubesinnung und Ausweg}

Die genannten Defizite des durch Richterrecht entfesselten Wirtschaftsstrafrechts stellen Rechtswissenschaft und Kriminologie vor neue Herausforderungen.

Einzufordern ist zunächst eine Kritik des Wirtschaftsstrafrechts aus kriminologischer Sicht. Die Kriminologie sollte ihr kritisches Potential auch im Rahmen einer Analyse des Wirtschaftsstrafrechts entfalten. Dies gilt zunächst für die Prozesse der Normgenese, der Auslegung des geltenden Rechts sowie deren Beeinflussung durch die mit Definitionsmacht ausgestatteten Akteure. Die obigen Ausführungen stellen diesbezüglich lediglich einen Anfang dar und beziehen sich auf ein eng umgrenztes Feld vornehmlich des Medizinstrafrechts. Diese Perspektive gilt es auf andere Regelungsmaterien des Wirtschaftsstrafrechts zu erweitern. Darüber hinaus wäre die Anwendung wirtschaftsstrafrechtlicher Normen auf unterschiedliche Gruppen von Normadressaten zu hinterfragen ${ }^{70}$, sowie die Bedeutung der Normen in der Praxis mit Blick auf mögliche Felder der Entkriminalisierung zu analysieren.

Rechtssoziologisch steht, abgesehen von der bemerkenswerten Arbeit von Beckemper $^{71}$, eine ökonomische Analyse des Wirtschaftsstrafrechts nach den von Posner entwickelten Maximen noch aus. Inhaltlich muss es insoweit darum gehen, die Kosten bestimmter wirtschaftsstrafrechtlicher Normen und Entscheidungen sowohl der Kriminalisierung als auch der Nicht-Kriminalisierung und Entkriminalisierung namhaft zu machen.

Aus strafrechtsdogmatischer Perspektive gilt es, der Neukriminalisierung durch Richterrecht Grenzen aufzuzeigen. Die Bedeutung der Strafrechtsbegrenzungsprinzipien der „ultima ratio“, des „Bestimmtheitsgrundsatzes“ und der „Subsidiarität“ sind für einzelne Rechtsfragen differenziert auszuloten $^{72}$. Diesbezüglich hat der Verfasser bereits an anderer Stelle exemplarisch vorgeschlagen $^{73}$, bei Vorliegen eines Leistungsaustauschs auf der Grundlage gegenseitiger Verträge den Vorteil i. S. der $\mathbb{S}$ 299, 331ff. $\mathrm{StGB}$ zu verneinen. Alleine diese Lösung vermeidet auch Eingriffe in die Vertragsfreiheit. Denn die gegenwärtige Interpretation des Vorteilsbegriffs macht Staatsanwälte und Richter zu Hütern der Angemessenheit der gezahlten Vergütung, die somit ohne strafrechtliche Risiken nicht mehr frei von den Vertragspartnern ausgehandelt werden kann. Dies setzt die marktwirtschaftliche Preisbildungstheorie in dem genannten Fallspektrum außer Kraft.

Eine vergleichbare teleologische Reduktion des Vorteilsbegriffs ist auch für solche Drittvorteile in Erwägung zu ziehen, die zu keinerlei Eigenvorteil des betroffenen Amtsträgers bzw. Normadressaten des $\ 299$ StGB geführt haben. Denn insoweit ist die Unparteilichkeit des Amtsträgers nicht gefährdet und damit das von $\mathbb{\int} \int 331 \mathrm{ff}$. StGB geschützte Rechtsgut nicht betroffen. Zur Veranschaulichung kann auf den Schulfotographenfall verwiesen werden. Die $\mathrm{Zu}$ wendungen an die Klassenkasse sind faktisch ein Rabatt, der auch den Kindern zugute kommt, die keine Bilder bestellt haben. Der Klassenlehrer hat davon nichts und er erweist sich in den Augen der Öffentlichkeit nicht als käuflich. Seine Kriminalisierung ist nicht angezeigt.

Statt avantgardistischer Strafrechtsdogmatik $^{74}$ mit akzessorischer Begriffsbildung und Prozeduralisierung plädiert der Verfasser dafür, die maßgeblichen Grenzen und Konturen der strafrechtlichen Tatbestandsmerkmale durch Auslegung der Tatbestandsvoraussetzungen selbst vorzunehmen ${ }^{75}$. Die Arbeit des Juristen ist und bleibt damit die bescheidene Auslegung des geltenden Rechts mit dem dafür vorgesehene Werkzeug der Gesetzeshermeneutik. Neue Kriminalpolitik gehört in die Hände des Gesetzgebers. 


\section{Fußnoten}

1 Scheerer: Der politisch-publizistische Verstärkerkreislauf. Zur Beeinflussung der Massenmedien im Prozess strafrechtlicher Normgenese, KrimJ 1978, 223 ff.; beispielhafte Verdeutlichung anhand des Diskurses über „Korruption im Gesundheitswesen" bei Schneider, in Boemke/Schneider: Korruptionsprävention im Gesundheitswesen, Düsseldorf 2011, S. $12 \mathrm{ff}$.

2 Sarbanes\&Oxley Act 2002; UK Bribary Act 2010. Überblick bei Klengel/Dymek: Criminal Compliance in den Zeiten des UK Bribary Act, HRRS 2011, 22ff.; zur Entwicklung der Gesetzgebung im Bereich des Wirtschaftsstrafrechts in Deutschland: Grunst/Volk, in: Volk (Hrsg.): Verteidigung in Wirtschafts- und Steuerstrafsachen, München 2006, S. 18ff.

3 Kempf/Lüderssen/Volk (Hrsg.): Die Handlungsfreiheit des Unternehmers - wirtschaftliche Perspektiven, strafrechtliche und ethische Schranken, Berlin 2009.

4 Schrödel: Aktuelle Erscheinungsformen der Wirtschaftskriminalität und Schwierigkeiten ihrer Bewältigung. Verteidigung in Wirtschaftsstrafsachen, in: Bannenberg/Jehle (Hrsg.): Wirtschaftskriminalität, Mönchengladbach 2010, S. $47 \mathrm{ff} ., 51 \mathrm{f}$

5 Paradigmatisch: Leipold/Tsambikakis/Zöller (Hrsg.): Anwaltskommentar StGB, Bonn 2011.

6 Z.B. Geiger: Nemo ultra posse obligatur. Zur strafrechtlichen Haftung von Compliance Beauftragten ohne Diszipinargewalt, CCZ 2011, 170ff.; ders.: Sponsoringverträge im Lichte des FSA-Kodex „Fachkreise“, A\&R 2009, $203 \mathrm{ff} .$.

7 Sickmüller/Breitkopf: „Points to Consider“ zu Anwendungsbeobachtungen. Empfehlungen des Bundesverbandes der Pharmazeutischen Industrie zur Durchführung von Anwendungsbeobachtungen, Pharm Ind 2009, 764ff.

8 Grundlegend: Eicker: Die Prozeduraliserung des Strafrechts. Zur Entstehung, Bedeutung und Zukunft eines Paradigmenwechsels, Bern 2010 .

9 Vgl, zum Beispiel die Kommentierung des \$2 299 StGB durch Diemer/Krick in: Münchener Kommentar zum Strafgesetzbuch, Band 4 \$S 263-358.

10 Vgl. Fischer: Strafgesetzbuch, 58. Aufl. 2011, \299 Rn. 10a.

11 Vgl. z.B. Bannenberg, in: NK Gesamtes Straf recht, 2. Aufl. 2011, \$ 299 Rn. 9; Kölbel: Strafbarkeitsnahe vertragsärztliche Kooperationsformen, NStZ 2011, 195ff. (wiederum zur Frage der Anwendung des $\mathbb{} 299$ StGB auf Vertragsärzte).

12 Ansätze bei Albrecht: Kriminologie. Eine Grundlegung zum Strafrecht, München, 4. Aufl. 2010, S. $322 \mathrm{ff}$.

13 Schneider: Wirtschaftskriminalität, Wirtschaftsstrafrecht und Wirtschaftskriminologie - Über die Erstarrung der deutschen Kriminologie zwischen atypischem Moralunternehmertum und Bedarfswissenschaft, in: Kempf/ Lüderssen/Volk (Hrsg.): Die Handlungsfreiheit des Unternehmers - wirtschaftliche Perspektiven, strafrechtliche und ethische Schranken, Berlin 2009, S. $61 \mathrm{ff}$.

14 Eindrucksvolle Beispiele aus der Gesetzgebung bei Nestler: Ökonomische Folgen verfehlter Kriminalisierung, in: Kempf/Lüderssen/Volk (Hrsg.): Die Handlungsfreiheit des Unternehmers - wirtschaftliche Perspektiven, strafrechtliche und ethische Schranken, Berlin 2009, S. $80 \mathrm{ff}$.

15 BGH, Urt. v. 17.07.2009, 5 StR 394/08, BGHS 54, 44ff., vgl. hierzu näher Schneider: Überwachungsgaranten in Unternehmen. Neue Probleme der Geschäftsherrenhaftung am Beispiel der Entscheidung des Bundesgerichtshofs vom 17. Juli 2009 - 5 StR 394/08, in: Kühl/Seher (Hrsg.): Rom, Recht, Religion. Festschrift für Udo Ebert, Tübingen 2011 (im Druck); ders./ Gottschaldt: Offene Grundsatzfragen der strafrechtlichen Verantwortlichkeit von Compliance-Beauftragten in Unternehmen, ZIS 2011, $573 \mathrm{ff}$
16 Lambrecht: Obiter dictum - Arabeske oder Balast, NJW 1998, 1039ff.

17 OLG Braunschweig, Beschl. v. 23.02.2010, Ws 17/10, StV 2010, 365f. mit Anmerkung Schneider, StV 2010, 366ff.; vgl. auch Schneider: Strafrechtliche Grenzen des Pharmamarketings. Zur Strafbarkeit der Annahme umsatzbezogener materieller Zuwendungen durch niedergelassene Vertragsärzte, HRRS 2010, $241 \mathrm{ff}$

18 BGH, Beschl. v. 05. Mai 2011, 3 StR 458/10; BGH, Beschl. v. 20. Juli 2011, 5 StR 115/11, vgl. hierzu näher: Schneider/Strauß: Die Zukunft der Anwendungsbeobachtungen. Rechtssichere Grenzen zwischen Korruption und zulässiger Kooperation angesichts der aktuellen Vorlagebeschlüsse des 3. und 5. Senats des Bundesgerichtshofs, HRRS 2011 (im Druck).

19 Hannich, in: Karlsruher Kommentar StPO, GVG, EGGVG, EMRK, $\mathbb{1 3 2}$ GVG Rn. 16.

20 BGH, Beschl. v. 05. Mai. 2011, 3 StR 458/10, Rn. 52, 53.

21 BGHSt 31, 264, 279f.; BGH wistra 2003, 303, 304; BGH NStZ 2005, 334, 335; 2008, 216 , 217.

22 BGH, Beschl. v. 26. Mai 2011, 3 StR 492/10.

23 Grundlegend: BGHSt 47, 295, 303ff.

$24 \mathrm{Vgl}$. vor allem Schlüter: Das obiter dictum, München 1973, S. 24ff., 191, der wegen des Übergriffs der Rechtsprechung in das Aufgabenfeld des Gesetzgebers die Gerichte dazu aufruft, zu ,einer vorsichtigen, mehr einzelfallbezogenen Begründungsweise zurückzukehren, wie sie auch heute noch in ihren Geschäftsordnungen vorgeschrieben ist".

25 Hirsch: Auf dem Weg zum Richterstaat? Vom Verhältnis des Richters zum Gesetzgeber in unserer Zeit, JZ 2007, 853ff.; Rüthers: Demokratischer Rechtsstaat oder oligarchischer Richterstaat, JZ 2002, 365ff.

26 Die für die Entscheidung verantwortlichen Richter und die beteiligten Staatsanwälte sind daher gern gesehene Referenten auf den einschlägigen Fortbildungsveranstaltungen, bei denen sie aber nicht selten von den Organisatoren als Marketinginstrumente im Rahmen des Geschäftes mit der Angst vor dem Strafrecht" instrumentalisiert werden. Eine juristische Beratungsleistung oder ein Compliance Produkt verkauft sich eben besser, wenn die Folgen von Noncompliance durch den Staatsanwalt oder Richter eindrucksvoll personifiziert werden.

27 Vgl. nur Lilie: Obiter dictum und Divergenzausgleich in Strafsachen, Köln u.a. 1993, S. 235, der zur Ausstrahlungswirkung von obiter dicta auf die Rechtsprechung der Instanzrichter schreibt: „Hier hat sich bislang die Tendenz nachweisen lassen, daß die vom Bundesgerichtshof gebrauchte Trennung zwischen ratio decidendi und obiter dictum von den Tatsacheninstanzen nicht immer mitgetragen wird.

Dabei scheint die Trennung zwischen ratio und dictum in den Sog hinsichtlich der Beurteilung der Präjudizienbindung mit hineingezogen worden zu sein. Beide Aspekte sind zu trennen".

28 Nach Lilie (Fn. 27), S. 250ff. könne es gar dahinstehen, ob es sich bei den Entscheidungsgründen der Strafsenate des Bundesgerichtshofs um tragende oder lediglich obiter dicta handele. Denn nur wenn alle in höchstrichterlichen Entscheidungen geäußerten Rechtsauffassungen als verbindlich erachtet werden, könne eine vorhersehbare und gleiche Rechtsanwendung garantiert werden, die es dem Bürger ermögliche, sein Handeln mit hinreichender Sicherheit zu beurteilen.

29 Näher: Albrecht (Fn. 12), S. $331 \mathrm{ff}$.

30 Schneider: Unberechenbares Strafrecht. Vermeidbare Bestimmtheitsdefizite im Tatbestand der Vorteilsannahme und ihre Auswirkungen auf die Praxis des Gesundheitswesens, in: Ders. u.a. (Hrsg.): Festschrift für Manfred Seebode zum 70. Geburtstag, Berlin 2008, S. 331ff., 334.

31 Die beiden Begriffe gehen zurück auf Steiner: Nach Babel. Aspekte der Sprache und des Übersetzens, Frankfurt am Main 1992, S. 129ff. Für den Hinweis auf Steiner danke ich Herrn Andreas Franke, FAZMED GmbH, Sonneberg.

32 Tag: Drittmitteleinwerbung - strafbare Dienstpflicht? Überlegungen zur Novellierung des Straftatbestands der Vorteilsannahme, JR 2004, 50, 52.

33 Der Bundestag geht in seiner Begründung zum Entwurf des Korruptionsbekämpfungsgesetzes explizit davon aus, dass die Erstreckung der Amtsträgereigenschaft auf die Beschäftigten privatrechtlich organisierter Rechtssubjekte im Bereich der öffentlichen Aufgabenwahrnehmung notwendig sei, um ,erhebliche Strafbarkeitslücken, insbesondere bei der Bekämpfung von Korruption“" zu schließen (BT-Drs. 13/5584, S. 12). Bzgl. \ 299 StGB vgl. BGH NJW 1968, 1572, 1573; Dannecker, in: NK StGB, 3. Aufl. 2010, \$299 Rn. 22; Tiedemann, in: LK StGB, 12. Aufl. 2008, $\mathbb{S} 299$ Rn. 16; Heine, in: Schönke/Schröder, StGB, 28. Aufl. 2010, \ 299 Rn. 8.

34 Vgl. zum Begriff des Beauftragten Fischer: Strafgesetzbuch, 58. Aufl. 2011, \$2 299 Rn. 10; Die mer/Krick, in: MüKo StGB, 2006, \299 Rn. 5 (m. w. N. zur Rechtsprechung): „Beauftragter ist jeder, der - ohne Inhaber oder Angestellter eines Betriebes zu sein - auf Grund seiner Stellung im Betrieb berechtigt und verpflichtet ist, für den Betrieb geschäftlich zu handeln, und unmittelbar oder mittelbar Einfluss auf die im Rahmen des Geschäftsbetriebes zu treffenden Entscheidungen ausüben kann. Der Begriff ist weit auszulegen." Danach sei die Beauftragung faktisch zu bestimmen und könne auch gerichtlich oder behördlich erfolgen; ein Rechtsgeschäft ist somit nicht zwingend vorausgesetzt Zum Begriff des Geschäftlichen Betriebes vgl. nur Bannenberg, in: NK Gesamtes Strafrecht, 2. Aufl. 2011, 299 Rn. 7: „Dieser Begriff ist sehr weit zu verstehen".

35 Vgl. Schneider (Fn. 30), S. 339ff.; Tag (Fn. 32), S. 53f.; dies./Tröger: Drittmitteleinwerbung strafbare Dienstpflicht?, Dtsch Ärztebl 2003 (100. Jg.), 2776, 2779 sowie die Kritik bei Esser: Strafrechtliche Relevanz der Drittmitteleinwerbung unter besonderer Berücksichtigung der Kooperation von Industrie und Ärzteschaft, Hamburg 2008, S. 152ff. m. w. N.

36 BGHSt 47, 295ff.

37 Näher: Schneider: Plädoyer für die Abkehr von der „streng formalen Betrachtungsweise“ im Bereich des Abrechnungsbetruges, in: Wienke/ Jahnke/Kramer (Hrsg.): Der Arzt im Wirtschaftsstrafrecht. Abkehr von unerwünschten und unerwarteten Strafbarkeitsrisiken in der vertragsärztlichen Berufsausübung, Heidelberg 2011, S. 57ff. m. w. N. zur Rechtsprechung.

38 Zurückhaltender: Tag (Fn. 32), S. 53.

39 Grundlegend: Lüderssen: Primäre oder sekundäre Zuständigkeit des Strafrechts?, in: Arnold u.a. (Hrsg.), Menschengerechtes Strafrecht. Festschrift für Albin Eser zum 70. Geburtstag, München 2005, S. 163ff., 174: „Der obrigkeitliche Zug bei der Anwendung der $\mathbb{S} \$ 331 \mathrm{ff}$ StGB kommt auch darin zum Ausdruck, dass die strafrechtliche Haftung nur beim Vorliegen entsprechender Genehmigungen ausgeschlossen sein soll - obwohl diese ja nichts dazu beitragen können, eine Drittmitteleinwerbung in der Substanz zu rechtfertigen und im Falle einer sozialadäquaten Drittmitteleinwerbung gar nich erforderlich sein dürften “. Später [S. 177] heißt es: „[Selbst] die ausdrückliche Bezugnahme eines Blankett-Strafgesetzes auf die zu Grunde liegende außerstrafrechtliche Norm kann nicht den Ausschlag geben. Von dieser Formalität kann nicht abhängen, was strafbar ist und was nicht. Vielmehr muss auch das interne Blankett - in dieser Funktion - den Anforderungen der Bestimmtheit genügen“. Vgl. auch Lüderssen Entkriminalisierung des Wirtschaftsstrafrechts II, Baden-Baden 2007, S. 145ff.

40 Lüderssen (Fn. 39), S. 170; beispielhafte Verdeutlichung bei Schneider/Gottschaldt (Fn. 15), S. 576f.; Schneider: Das Strafrecht als Büttel des Sozialrechts? Ein Plädoyer für die Autonomie strafrechtlicher Begriffsbildung im Fall des 
Abrechnungsbetruges im Gesundheitswesen, in: Duttge (Hrsg.): Tatort Gesundheitsmarkt: Rechtswirklichkeit - Strafwürdigkeit - Prävention, Göttingen 2011 (im Erscheinen); Theile: Konvergenzen und Divergenzen zwischen Gesellschaftsrecht und Strafrecht - Das Merkmal der gravierenden Pflichtverletzung ( $\$ 266$ StGB) aus systemtheoretischer Sicht, ZIS 2011 , 616, 626f.

41 Popitz: Die normative Konstruktion von Gesellschaft, Berlin 1980, S. 41.

42 Michels: Weiße Kittel - Dunkle Geschäfte. Im Kampf gegen die Gesundheitsmafia, Berlin 2009.

43 Michels (Fn. 42), S. 74f., $196 \mathrm{ff}$

44 Michels (Fn. 42), S. 74: „Bei den Krebsvorsorgeuntersuchungen vertraten die Strafverfolger die Ansicht, der Gynäkologe habe nicht vorsätzlich gehandelt. Wenigstens führte unsere Beschwerde dazu, dass weiter ermittelt wurde. Denn wichtig ist: Wenn ein Arzt immer nur die falsch abgerechneten Beträge erstatten muss und sonst nichts zu befürchten hat, wird ihn auch künftig nichts davon abhalten, weiterhin Abrechnungen zu frisieren“.

45 http://www.transparency.de/Die-Gruendungsgeschichte-von-T.1420.0.html (zuletzt besucht: 06.09.2011).

46 „Transparency Deutschland arbeitet nicht konfrontativ. Um Veränderungen voranzutreiben, bilden wir Koalitionen mit denjenigen Regierungen, Verwaltungen, Politikern, Firmen und zivilgesellschaftlichen Gruppen, die eine vertrauenswürdige, transparente, werteorientierte und zivile demokratische Politikkultur vertreten“. http://www.transparency. de/Standpunkte.43.0.html (zuletzt besucht: 06.09.2011).

47 Vgl. z.B.: http://www.transparency.de/Berichtvon-der-European-Heal.630.0.html (zuletzt besucht 06.09.2011): „Von den rund tausend Milliarden (= 1 Billion) Euro, die in Europa jährlich im Gesundheitsmarkt ausgegeben werden, entstehen zwischen dreißig und einhundert Milliarden Verlust durch Betrug und Korruption. Diese Zahl wurde analog zu amerikanischen Erfahrungen zugrunde gelegt, denn dort werden 3-10\% des gesamten Gesundheitsbudgets durch Betrug und Korruption aufgezehrt. In Europa sind die Zustände im Prinzip nicht besser".

48 TI definiert Korruption als „Missbrauch von anvertrauter Macht zum privaten Nutzen oder Vorteil“, vgl. hierzu Ziercke: Die internationale Bekämpfung der Korruption und die Rolle des Bundeskriminalamtes, in: TI/Friedrich Ebert Stiftung (Hrsg.): Strafverfolgung der Korruption 2010 - „Neue“ Strategien der Korruptionsbekämpfung, Berlin 2010, S. 7ff.

49 Paradigmatisch: Spelsberg: Rezension zu Boemke/Schneider: Korruptionsprävention im Gesundheitswesen, in: Scheinwerfer 52, Juli 2011, S. 36: „Die Autoren vertreten dabei zum Teil Auffassungen, die nachhaltige Korruptionsprävention verhindern“.
50 Z.B. Positionspapier zur Reform des Straftatbestands der Bestechung im geschäftlichen Verkehr: http://www.transparency.de/filead$\mathrm{min} / \mathrm{pdfs} /$ Themen/Wirtschaft/\$299\%20-\%20 Positionspapier_09-09-30.pdf (zuletzt besucht: 06.09.2011), vgl. zu einem Gesetzesentwurf der SPD ferner http://www.transparency.de/Transparency-schafft-es-in-den.1951.0.html (zuletzt besucht: 06.09.2011): „Und wie das Kaninchen auf die Schlange schaut die Regierung auf den Bundesgerichtshof, der demnächst entscheiden wird, ob niedergelassene Ärzte wie Amtsträger behandelt werden dürfen. Anstatt den üblichen Rundumschlag zu landen, die Opposition diskriminiere einen ganzen Berufsstand, sollte die Regierungskoalition die Möglichkeit nutzen, dem Bundesgerichtshof zuvor zu kommen und niedergelassene Ärzte im Hinblick auf Vorteilnahme und Bestechlichkeit den Krankenhausärzten gesetzlich gleichzustellen “.

51 Vgl. das Referat bzw. die Publikation des Staatsanwalts André Schmidt: Kassenärzte als taugliche Täter in Bestechungsdelikten im geschäftlichen Verkehr - Beschluss des OLG Braunschweig vom 23.02.2010, in: TI/Friedrich Ebert Stiftung (Hrsg.): Strafverfolgung der Korruption 2010 - „Neue“ Strategien der Korruptionsbekämpfung, Berlin 2010, S. $79 \mathrm{ff}$. Der im Übrigen sehr abgewogen und kundig argumentierende StA Schmidt war zuständig im Verfahren vor dem OLG Braunschweig.

52 Vgl. z.B. die "Stellungnahme von Transparency International Deutschland e.V. zum Entwurf eines Gesetzes zur Regelung des Beschäftigtendatenschutzes (Stand des Gesetzesentwurfs: 28.05.2010); http://www.transparency.de/ fileadmin/pdfs/Themen/Wirtschaft/Stellungnahme_BDSG_10-06-28.pdf (zuletzt besucht 06.09.2011); ähnlich_Michels (Fn. 42), S. 201: „Datenschutz darf nicht länger Täterschutz sein".

53 Bonger: Criminality and Economic Conditions, 1916.

54 Coleman: The criminal elite. Understanding white-collar crime, New York 2006, S. 198; ähnlich Taylor: Crime in Context. A critical Criminology of market society, Boulder 1999.

55 Shover/Hochsteler, Choosing White-Collar Crime, 2006; Shover'́Grabosky, WhiteCollar crime and the Great Recession, Criminology \&Public Policy 2010, 429 ff.; Grabosky/Shover, Forestalling the next epidemic of white-collar crime. Linking policy to theory, Criminology\&Public Policy 2010, 641ff.

56 Vgl. die in Fußnote 55 genannten Arbeiten.

57 Vgl. Coleman (Fn. 54), S. 204: To create "a stigmatizing label for those who violate its standards - a label that is, moreover, especially repugnant to the respectable businesspeoble, politicians, and the professionals who constitute the majority of white-collar criminals".

58 Schneider (Fn. 13), S. $63 \mathrm{ff}$.

59 Schneider/John: Der Wirtschaftsstraftäter in seinen sozialen Bezügen. Empirische Befunde und Konsequenzen für die Unternehmenspra- xis, in: Bannenberg/Jehle (Hrsg.): Wirtschaftskriminalität, Mönchengladbach 2010, S. $159 \mathrm{ff}$

60 Cleff/Luppold/Naderer/Volkert, in: PricewaterhouseCoopers (Hrsg.): Wirtschaftskriminalität. Eine Analyse von Motivstrukturen, 2009; Zusammenfassung bei Salvenmoser/Schmitt: Persönlichkeit von Wirtschaftsstraftätern: Eine empirische Studie, in: Bannenberg/Jehle, Wirtschaftskriminalität, Mönchengladbach 2010, S. $151 \mathrm{ff}$.

61 Cleff/Luppold/Naderer/Volkert (Fn. 60), S. 33

62 Laue: Die konventionelle Theorie als Grundlage der Wirtschaftskriminologie und des Wirtschaftsstrafrechts, in: Bannenberg/Jehle (Hrsg.): Wirtschaftskriminalität, Mönchengladbach 2010, S. $117 \mathrm{ff}$.

63 Eicker (Fn. 8), $21 \mathrm{f}$.

64 Kritisch zu Ausweitungstendenzen im Strafrecht auch: Prittwitz: Das deutsche Strafrecht: Fragmentarisch? Subsidiär? Ultima ratio?, in: Institut für Kriminalwissenschaften Frankfurt a. M. (Hrsg.): Vom unmöglichen Zustand des Strafrechts, Frankfurt a. M. u.a. 1995 , S. 387ff., 405: „Es steht fest, daß wir nur begrenzte Ressourcen haben und für Strafrecht einsetzen wollen. Es spricht viel dafür, daß das Mittel Strafrecht sich abnutzt, wenn es inflationär eingesetzt wird“"

65 Ausführlich zu diesen Mechanismen der positiven Generalprävention: Schneider (Fn. 13), S. 61ff. Die rechtssozilogischen Grundüberlegungen sind dargelegt bei Popitz (Fn. 41).

66 Eingehend: Schneider/Strauß (Fn. 18).

67 Lieb/Brandtönies: A Survey of German Physicians in Private Practice About Contacts With Pharmaceutical Sales Representatives, Dtsch Ärztebl Int 2010, 392, 394.

68 Schneider (Fn. 13), S. 72f.; ders. (Fn. 40).

69 Schneider (Fn. 30), S. 338.

70 Wegweisend: Homann: Betrug in der gesetzlichen Krankenversicherung. Eine empirische Untersuchung über vermögensschädigendes Fehlverhalten zulasten der Solidargemeinschaft, Mönchengladbach 2009.

71 Beckemper: Ökonomische Analyse der Täuschungsdelikte des Wirtschaftstrafrechts, Potsdam, unveröffentlichte Habilitationsschrift.

72 Beispielhaft: Hamm: Begrenzung des Wirtschaftsstrafrechts durch die Grundsätze der ultima ratio, der Bestimmtheit der Tatbestände, des Schuldgrundsatzes, der Akzessorietät und der Subsidiarität, in: Kempf/Lüderssen/ Volk (Hrsg.): Die Handlungsfreiheit des Unternehmers - wirtschaftliche Perspektiven, strafrechtliche und ethische Schranken, Berlin 2009 , S. 44ff.; Schneider (Fn. 30), S. 331ff.; Theile (Fn. 40), 616ff.

73 Schneider (Fn. 30), S. 347ff.

$74 \mathrm{Zu}$ diesem Begriff näher: Schneider/Morguet: Gefährliches Strafrecht. Zu den Grenzen avantgardistischer Strafrechtsdogmatik, in: Uwer (Hrsg.): Bitte bewahren sie Ruhe. Leben im Feindrechtsstaat, Berlin 2006, S. $335 \mathrm{ff}$.

75 Schneider (Fn. 37), S. 57ff.; ders. (Fn. 40).

\section{Dreiländertagung „Wegsperren - und zwar für immer?“ 4. / 5. Juni 2012 in Luzern, Schweiz}

Resozialisierung im Spannungsfeld von Sicherheitserwartungen und sozialer Integration: Standortbestimmung und neue Perspektiven in der Schweiz, in Deutschland und Österreich

Spätestens seit den 1970er- Jahren galt in demokratischen Rechtsstaaten wie in der Schweiz, in Deutschland oder in Österreich die Resozialisierung als zentrales und unbestrittenes Ziel des Strafvollzugs. Resozialisierung und Bewährung gehören denn auch nach wie vor zu den primären Zielsetzungen des Straf- und Massnahmenvollzugs in den gesetzlichen Grundlagen dieser Länder. Heute ist hingegen die öffentliche Diskussion über Strafjustiz und Strafvollzug geprägt von Forderungen nach Nulltoleranz, Sicherheit und Wegsperren von gefährlichen Straftätern. Es stellt sich deshalb eine Reihe von Fragen: Ist die Idee der Resozialisierung gescheitert? Kann gesellschaftliche Sicherheit allein durch harte, unerbittliche Strafen hergestellt werden? Und wie sehen die Perspektiven von Resozialisierung und Sicherheit im nationalen und internationalen Vergleich aus?

An der internationalen Fachtagung „Wegsperren - und zwar für immer?“ vom 4./ 5 Juni 2012 in Luzern versuchen anerkannte Fachleute aus Deutschland, Österreich und der Schweiz auf diese Fragen eine Antwort zu geben. Mehr dazu auf: www.hslu.ch/dreilaendertagung 